\title{
Das fiebernde Kind in der pädiatrischen Notaufnahme
}

\section{Gesche Kranz}

Wenn ein Kind in die Notaufnahme kommt, ist der häufigste Grund dafür Fieber.

Für die erhöhte Körpertemperatur können viele Faktoren verantwortlich sein. In der

Ersteinschätzung gilt es, Kinder mit besonderem Behandlungsbedarf zu identifizieren eine große Herausforderung für die Pflege.



Hohes Fieber bei Säuglingen ist für Eltern immer ein Alarmsignal - dabei sagt die Höhe nicht immer etwas über die Behandlungsdringlichkeit aus. (Symbolbild/Quelle: artem_goncharov/stock.adobe.com)

Seit Jahren steigen die Patientenzahlen in Notaufnahmen. Dies ist kein speziell deutsches Problem, sondern zeigt sich international. Die Inanspruchnahme findet vermehrt durch Patienten statt, die aus professioneller Sicht nicht als Notfall einzustufen sind [1]. Aufgrund der hohen
Patientenzahlen ist eine Sortierung der Patienten nach Behandlungsdringlichkeit unabdingbar. Der folgende Beitrag betrachtet die Herausforderung dieser Einschätzung anhand eines der häufigsten Vorstellungsgründe in Kindernotaufnahmen: das fiebernde Kind. 
Ein häufiger Vorstellungsgrund für Kinder in Notaufnahmen ist Fieber. Teilweise können die Angehörigen keine Begleitsymptome nennen. Sie können sich das Fieber nicht erklären, dem Kind würde es sonst eigentlich gut gehen. Die Dauer des Fieberauftretens variiert zwischen „hat gerade angefangen“ bis zu „Mein Kind fiebert jetzt seit zehn Tagen“. Die Eltern präsentieren sich in jedem Fall immer besorgt um den Zustand des Kindes und in Angst vor einer schweren Erkrankung. Diese Sorge trifft auf die Problematik der Dringlichkeitseinschätzung in Notaufnahmen, da sich die Sicht des medizinischen Fachpersonals auf das fiebernde Kind häufig vom Eindruck der Eltern unterscheidet.

\section{Wann ist es ein Notfall?}

Um zu verstehen, warum Eltern durchaus verständlicherweise den Weg in die Notaufnahme suchen, während das medizinische Fachpersonal die Dringlichkeit einer Behandlung als nicht so hoch einschätzt, ist es wichtig zu wissen, wann es sich um einen Notfall handelt. Behringer et al. geben folgende Definition für den Begriff Notfallpatienten aus:

„Als medizinischer Notfall bzw. als Notfallpatienten werden alle Personen definiert, die körperliche oder psychische Veränderungen im Gesundheitszustand aufweisen, für welche der Patient selbst oder eine Drittperson unverzügliche medizinische und pflegerische Betreuung als notwendig erachtet. " [2]

Hier wird die subjektive Einschätzung der Patienten selbst als einschätzender Faktor genannt. Dabei kann die subjektive Beurteilung der Dringlichkeit einer Behandlung zwischen verschiedenen Patienten, abhängig von Faktoren wie Alter, Geschlecht oder Bildungsgrad, zum Teil sehr unterschiedlich ausfallen [3]. Eine andere häufig genutzte Definition für Notfallpatienten, die im Hessischen Rettungsdienstgesetz $\S 3$ Abs. 4 zu finden ist, sagt:

„Notfallpatientinnen oder Notfallpatienten sind Personen, die sich infolge einer Erkrankung, Verletzung, Vergiftung oder aus sonstigen Gründen in unmittelbarer Lebensgefahr befinden oder bei denen diese zu erwarten ist oder bei denen schwere gesundheitliche Schäden zu befürchten sind, wenn keine schnellstmögliche notfallmedizinische Versorgung oder Überwachung und gegebenenfalls eine Beförderung zu weiterführenden diagnostischen oder therapeutischen Einrichtungen erfolgt.“

Die in diesem Fall abzuwägende Lebensgefahr ist häufig nur durch medizinisches Fachpersonal einschätzbar und müsste generell zu einer Behandlung in einem Krankenhaus führen [4]. Ärzte, Pflegekräfte und Rettungssanitäter nutzen für diese Beurteilung nicht nur ihren Erfahrungsschatz, sondern versuchen ihr subjektives Empfinden durch objektive Bewertungskriterien wie zum Beispiel die Erhebung von Vitalwerten zu verifizieren. Grundsätzlich ist aber festzuhalten, dass das subjektive Empfinden eines Betroffenen

\section{FALLBEISPIEL}

Donnerstagabend, 19.30 Uhr, Kindernotaufnahme: Der 16 Monate alte Julius* wird von seinen Eltern in der Notaufnahme vorgestellt. Er hat seit gestern Abend Fieber, zunächst um die $38,8^{\circ} \mathrm{C}$. Heute Morgen war die Mutter mit ihm beim Kinderarzt. Dieser hat Paracetamol verschrieben und etwas von einem roten Hals erzählt. Der Grund für die jetzige Vorstellung ist, dass das Fieber zuletzt bis $40,5^{\circ} \mathrm{C}$ gestiegen ist. Die Eltern haben heute zum dritten Mal ein fiebersenkendes Zäpfchen gegeben und meinen, dass es aber „eigentlich gar nichts nützt. Das Fieber geht nicht runter, sondern steigt nur noch mehr.“ Die Eltern sind in großer Sorge. Julius ist wach, interessiert und neugierig. Laut den Eltern trinkt er gut, essen will er aber nicht so richtig. Die rektale Temperaturmessung zeigt in der Triage $39,6^{\circ} \mathrm{C}$ an, ca. $1 \mathrm{~h}$ nach Paracetamolgabe.

* Name geändert

bzw. Nebenstehenden als Beurteilungsmaßstab gilt. Sehen diese eine unmittelbare Behandlung als nötig an, handelt es sich aus deren Sicht um einen Notfall. Das führt zwangsläufig dazu, dass das Patientenaufkommen in Notaufnahmen kaum planbar ist. Weder die Anzahl der sich gleichzeitig in der Notaufnahme Befindenden, noch die Schwere ihrer Symptome bzw. Erkrankungen ist derzeit steuerbar. Da nicht alle Patienten direkt und gleichzeitig behandelt werden können, ergibt sich die Notwendigkeit, Notfallpatienten mit lebensbedrohlichen Erkrankungen bei der Vorstellung in der Notaufnahme innerhalb kürzester Zeit zuverlässig zu erkennen [5][6]. Um dies zu gewährleisten, setzt sich in Deutschland mehr und mehr eine Einschätzung der Behandlungsdringlichkeit mittels einer strukturierten Triage durch. Sie dient in Notaufnahmen der Einschätzung des Schweregrads von Symptomen einer Erkrankung oder Verletzung, um damit eine Kategorisierung und Priorisierung der Behandlungsreihenfolge vornehmen zu können [6]. Außerdem soll die Triage helfen, die Patienten an den Ort zu leiten, der für ihre Behandlung die notwendigen Ressourcen vorhält [7]. Es gibt die verschiedensten Triage-Systeme. Als Goldstandard in der Notfallmedizin gelten diejenigen Einschätzungsinstrumente, die die Dringlichkeit mithilfe einer fünfstufigen Einteilung bewerten [5][6].

\section{Ersteinschätzung obliegt der Pflege}

Das in Deutschland verbreitetste Triage-System ist das Manchester-Triage-System. Hier ist vorgesehen, dass geschulte Pflegekräfte die Dringlichkeitseinschätzung vornehmen sollen, wie es auch internationaler Standard ist. Schon vor der Einführung der standardisierten TriageInstrumente in Notaufnahmen in Deutschland war die Ersteinschätzung des Behandlungsbedarfs bei Ankunft der Patienten in der Notaufnahme Aufgabe von Pflegekräften. 
Diese Aufgabenübertragung wurde auch bei der Umsetzung der strukturierten Triage nicht verändert, da die Erfahrung zeigte, dass die Pflegekräfte aufgrund ihrer Fachkompetenz und ihres breit angelegten Fachwissens hier gute Entscheidungen trafen [6].

Im Manchester-Triage-System wird die Körpertemperatur als genereller Indikator geführt. Das bedeutet, dass sich dieses Merkmal in allen Präsentationsdiagrammen der Hauptbeschwerden wiederfindet [8]. Das Einschätzungskriterium Temperatur wird eingeteilt in „sehr heiß“ $\left(>41,0^{\circ} \mathrm{C}\right)$, „heiß“ $\left(>38,5^{\circ} \mathrm{C}\right)$ und „erhöhte Temperatur“ $\left(>37,5^{\circ} \mathrm{C}\right)$ sowie „unterkühlt“" $\left(<35,0^{\circ} \mathrm{C}\right)$. Das Manchester-Triage-System unterscheidet die Einstufung von „fiebernden“ Patienten außerdem nach Altersgruppen ( $\$$ Tab. 1).

In Stufe 2 fällt ein Patient ab älter als ein Jahr mit der Einschätzung "sehr heiß“ (>41 $\mathrm{C}$ ). Ein Säugling (bis einschließlich 12. Lebensmonat) wird ab einer Temperatur von $38,5^{\circ} \mathrm{C}$ als sehr dringend in Stufe 2 eingeordnet. Das „überwärmte“ Neugeborene bis zum 28. Lebenstag wird in Dringlichkeitsstufe 3 eingestuft. Patienten ab dem zweiten Lebensjahr mit einer Körpertemperatur $>38,5^{\circ} \mathrm{C}$ sowie überwärmte Patienten gehören in die Stufe 3 bzw. 4 und erhalten damit eine dringende bzw. normale Wartezeit bis zur Behandlung durch den Arzt [8].

Wie oben beschrieben stellen sich viele Patienten mit dieser Symptomatik vor. Es können allerdings nicht alle gleichzeitig dem Arzt präsentiert werden. Die Herausforderung für die Pflegekraft besteht also darin einzuschätzen, welche Patienten schwerer erkrankt sind und schneller einen Arztkontakt benötigen.

\section{Was ist Fieber?}

Eine genauere Betrachtung des Phänomens Fieber zeigt, dass es sich nicht um eine zu behandelnde Krankheit handelt, sondern um ein Symptom, das einen Hinweis auf eine Erkrankung gibt. Grundsätzlich ist Fieber „ein charakteristisches Symptom der Aktivierung des Immunsystems und der damit verbundenen Inflammationsreaktion “ [9] und ist Folge einer Zytokin-vermittelten Sollwerterhöhung im hypothalamischen Wärmeregulationszentrum. Es ist zu unterscheiden von der benignen Hyperthermie, die bei von außen zugeführter Wärme wie zum Beispiel Sonneneinstrahlung, zu warmer Kleidung oder körperlicher Aktivität entstehen kann. Bei der benignen Hyperthermie ist es typisch, dass die Körpertemperatur rasch wieder abfällt, sobald die zur Erhöhung führenden Umstände geändert werden [10].

Es gibt keine einheitliche Definition, ab wann von Fieber gesprochen wird, grundsätzlich scheint aber Einigkeit darüber zu bestehen, dass ab einer Körpertemperatur $>38,3^{\circ} \mathrm{C}$ leichtes bis mäßiges Fieber vorliegt. Teilweise liegt die begriffsbestimmende Grenze auch schon bei
- Tab. 1 Behandlungsdringlichkeit anhand des Indikators Temperatur.

\begin{tabular}{|l|l|l|}
\hline Einschätzungskriterium & Einstufung & Behandlungsdringlichkeit \\
\hline $\begin{array}{l}\text { Sehr heiß } \\
\text { Heißer Säugling } \\
\left(>38,5^{\circ} \mathrm{C}, \text { Lebensalter<1 Jahr) }\right. \\
\text { Unterkühlt }\end{array}$ & Stufe 2 & $\begin{array}{l}\text { Sehr dringend (Arztkontakt } \\
\text { spätestens nach 10 min) }\end{array}$ \\
\hline $\begin{array}{l}\text { Überwärmtes Neugeborenes } \\
\left(>37,5^{\circ} \mathrm{C}, \text { Lebensalter bis }\right. \\
28 \text { Tage) } \\
\text { Heiß }\end{array}$ & Stufe 3 & $\begin{array}{l}\text { Dringend (Arztkontakt } \\
\text { spätestens nach 30 min) }\end{array}$ \\
\hline $\begin{array}{l}\text { Überwärmt } \\
\end{array}$ & Stufe 4 & $\begin{array}{l}\text { Normal (Arztkontakt spä- } \\
\text { testens nach 90 min) }\end{array}$ \\
\hline
\end{tabular}

$38,0^{\circ} \mathrm{C}$, abhängig von bestimmten Vorrausetzungen wie etwa dem Alter des Kindes [11]. Für die Beurteilung der Körpertemperatur ist es wichtig, dass diese über den Tag verteilt physiologischen Schwankungen unterliegt, die zwischen $35,8^{\circ} \mathrm{C}$ und $38^{\circ} \mathrm{C}$ liegen können, mit einem zu erwartenden Maximum am Nachmittag. Schließlich haben auch noch Aspekte wie Lebensalter, körperliche Aktivität und immunologische sowie endokrine Einflüsse Auswirkungen auf die Körpertemperatur [9].

Neben der Interpretation der Körpertemperatur ist es entscheidend, wie die Körpertemperatur gemessen wird bzw. gemessen werden kann. Traditionell am bekanntesten sind die Varianten axillar, rektal und sublingual. Inzwischen setzen sich aber auch immer mehr die Methoden der Messung im Ohr oder an der Stirn durch. Alle Varianten haben ihre Vor- und Nachteile.

Bisher wurde die rektale Fiebermessung als die genaueste Ermittlung der Körperkerntemperatur bevorzugt. Aufgrund der bestehenden anderen Möglichkeiten zeigt sich jedoch immer mehr, dass diese Methode bei den Eltern eher unbeliebt ist, ausgelöst durch die Wehrhaftigkeit ihrer Kinder. Zudem gibt es diverse nicht vernachlässigbare Kontraindikationen, wie Blutungsneigung, Zustand nach analchirurgischen Eingriffen oder lokale Entzündungen.

Die Messung der Temperatur des Trommelfells kommt der Körperkerntemperatur durchaus nahe, womit sich das Ohrthermometer als genaueste Alternative anbietet [9]. Dabei ist allerdings die richtige Handhabung entscheidend. Die unkorrekte Lage des Ohrtrichters, ein verkrümmter Gehörgang oder Verlegung durch Cerumen können zu Fehlmessungen führen und vor allem potenziell hohe Temperaturen unterschätzen [11]. Die Akzeptanz dieser Methode ist aber sowohl bei Eltern als auch bei Kindern deutlich höher [12].

Die sublinguale oder bukkale Messung liegt durchschnittliche $0,6^{\circ} \mathrm{C}$ unter der rektalen Vergleichsmessung, wobei 


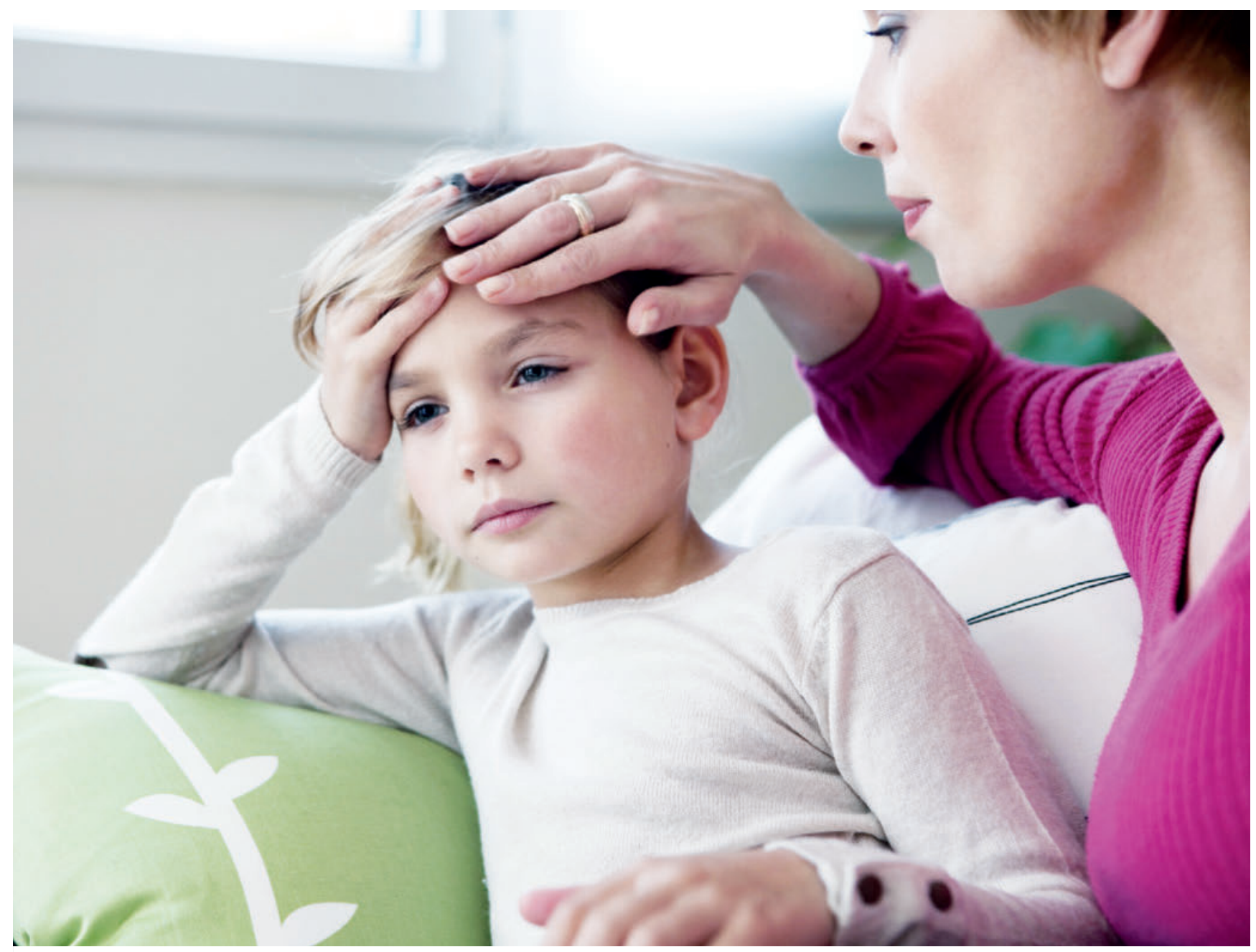

Neben Fieber ist der Allgemeinzustand eines Kindes ausschlaggebend dafür, ob dringend eine Behandlung erforderlich ist oder nicht. (Symbolbild/Quelle: RFBSIP/stock.adobe.com)

auch hier die richtige Anwendung ausschlaggebend ist. Das Messen in der Mundhöhle funktioniert nur bei verständigen kooperationsfähigen Patienten. Das vorherige Trinken von kalten oder warmen Getränken oder auch das bei verlegter Nasenatmung ständige Atmen durch den Mund führen zu Messwertverfälschungen.

Die axillare Temperaturbestimmung ist nach wie vor die bevorzugte Methode bei Neugeborenen oder auch Patienten mit Neutropenie [9]. Auch hier muss die nötige Compliance vorliegen, da sonst die Umgebungstemperatur Einfluss auf das Messergebnis haben und es zu falschen niedrigen Messwerten kommen kann.

Die am wenigsten störende Maßnahme für den Patienten ist die Messung der Stirntemperatur. Doch auch diese Messung ist ungenau, denn die Hauttemperatur an der Stirn kann bis zu $1^{\circ} \mathrm{C}$ unter der Kerntemperatur liegen, und es gibt Störfaktoren wie Umgebungstemperatur und feuchte schwitzige Haut [13].

\section{Das Gespräch mit den Eltern ist wichtig}

Diese verschiedenen Betrachtungsweisen bei der Einschätzung einer erhöhten Körpertemperatur (physiologische Gesichtspunkte, Wann beginnt Fieber? und Messverfahren) sind den Eltern oft gar nicht bewusst und können bei der Anamneseerhebung während der Triage bereits unterstützend helfen, die Sorge der Eltern zu mildern. Während des Erstkontakts zwischen Pflegekraft und Vorstellendem sollte ein Abgleich dieser Aspekte stattfinden, um die ersten Fehlerquellen wie missverständliches Annehmen von Fieber $\left(<38,0^{\circ} \mathrm{C}\right)$ durch die Angehörigen sowie fehlerhafte Messung und Interpretation des gemessenen Werts auszuschließen.

Entscheidend ist, in der Beurteilung des fiebernden Kindes kein schwer krankes Kind zu übersehen. Ausschlaggebend ist hierbei allerdings nicht die Höhe der Temperatur, sondern der Allgemeinzustand des Kindes. In Deutschland gibt es hierfür bisher keine festgesetzten Leitlinien, sondern es zählt die erfahrene Krankenbeobachtung der triagierenden Pflegekraft. Diese beurteilt neben der Höhe 
der Temperatur die Agilität des Kindes, die Reaktion auf die Angehörigen, die Hautfarbe sowie Hautausschläge, die Atmung und die Hydratation. Die Einschätzung ist vergleichbar mit dem Blickdiagnosedreieck (pediatric assesment triangle) der American Academy of Pediatrics zur Entscheidung, ob das Kind krank oder schwer krank ist. Hierbei sollen Allgemeinzustand, Atmung/Atemarbeit und Hautkolorit/-perfusion mit einem Blick überprüft werden [14].

Ist das Kind agil, kommuniziert mit den Eltern, ist gut erweckbar zur Triage, hat kein auffälliges Hautkolorit, atmet normal und zeigt sich im Allgemeinen entsprechend seines Alters, wird zunächst eine Beruhigung der Angehörigen angestrebt. Es gibt durchaus Kinder, die trotz Temperaturen von mehr als $39,0^{\circ} \mathrm{C}$ spielend im Wartezimmer umhertollen, von den Eltern auch gern präsentiert mit den Worten: „Man sieht unserem Kind nie an, dass es so krank ist." In dieser Situation sollte mit den Eltern besprochen werden, ob bei Temperaturen über $39,0^{\circ} \mathrm{C}$ eine Fiebersenkung sinnvoll ist oder nicht, denn wie oben beschrieben ist nicht das Bekämpfen des Fiebers das Ziel, sondern die Behandlung des Fieberauslösers. Fiebersenkung sollte hauptsächlich zur Verbesserung des Allgemeinzustands dienen, um den physiologischen Nutzen der Temperaturerhöhung wie eine erhöhte Immunantwort nicht zu stören. Bei Temperaturen über $40^{\circ} \mathrm{C}$ ist eine Fiebersenkung indiziert, da diese die Kinder stark erschöpfen können. Während der Fieberphase ist eine ausreichende Hydrierung der Kinder wichtig [11].

Gibt es bei einem der oben genannten Einschätzungskriterien Auffälligkeiten, ist eine erhöhte Aufmerksamkeit in der Triage geboten. Jedes Beobachtungsmerkmal kann entscheidend dafür sein, ob ein sofortiger Arztkontakt geboten ist oder das Kind zumindest in eine engmaschige Überwachung und Kontrolle durch das medizinische Fachpersonal übergehen sollte, bis zum Beginn der ärztlichen Diagnostik und Therapie.

\section{Eine Richtlinie aus Großbritannien kann helfen}

Obwohl nicht offiziell als Richtlinie in Deutschland ausgegeben, können hier die NICE-Guidelines einen guten Überblick über die Warnsignale und ihre Ausprägungen geben (online unter bit.ly/2BvHsAW, S.19-20). Diese werden in Großbritannien genutzt und sollen sowohl den Eltern und Betreuungspersonen als auch dem medizinischen Fachpersonal Hilfestellung in der Einschätzung des fiebernden Kindes geben. Hier werden die oben genannten Beobachtungsmerkmale erfasst und je nach Ausprägung in eine grüne Stufe mit geringem Risiko, eine gelbe Stufe mit mittlerem Risiko und eine rote Stufe mit hohem Risiko eingeteilt. Diese farbliche Unterteilung ist zu unterscheiden von der Einstufung der Manchester Triage. Die NICE-Guidelines geben keine zeitlichen Vorgaben zum Arztkontakt, son- dern sollen helfen, dass kritisch kranke Kind anhand von begleitenden Symptomen zu identifizieren und das weitere Handeln festzulegen [15].

Schon während des Erfragens des Vorstellungsgrunds ist es möglich, die Hautfarbe des Kindes einzuschätzen. Bei blassen Kindern ist es wichtig zu ermitteln, welcher Grund für die Blässe vorliegt. Ist die Hautfarbe marmoriert, blau oder grau, ist eine sofortige Monitorüberwachung indiziert und ein Arzt erforderlich, da dies ein Zeichen für eine Sepsis sein kann. Oft wird die Hautfarbe im Vergleich mit der Hautfarbe der Eltern betrachtet. Dies muss mit großer Vorsicht geschehen, um die Gefahr einer Fehlbeurteilung zu vermeiden. Oft ist es hilfreich, die Eltern zu befragen, ob sie Auffälligkeiten an der Hautfarbe sehen. Ein nicht wegdrückbarer Hautausschlag sollte zu sofortigem Arztkontakt führen, mit Verdacht auf Petechien, die ein Hinweis auf eine Meningokokken-Sepsis mit Verdacht auf ein Waterhouse Friderichsen Syndrom sein könnten.

Zeigt ein fieberndes Kind eine auffällige Atmung, sollte es mindestens in eine engmaschige Beobachtung der Pflegefachperson übergehen. Dazu gehört die Überprüfung der Sauerstoffsättigung, und bei deutlich angestrengter Atmung mit erhöhter Atemarbeit ist ein Arzt hinzuzuziehen, um eventuell mit Inhalationsmaßnahmen oder auch mit zusätzlicher Sauerstoffgabe zu unterstützen. Insbesondere die Veränderung der Atemarbeit nach Fiebersenkung ist von Interesse. Eine erhöhte Atemfrequenz kann hierdurch schon behoben sein.

Die Herzfrequenz ist als Beurteilungsmaßstab schwierig, vor allem wenn sich das Kind während der Triage sehr wehrhaft zeigt. Eine Temperatur über $39,0^{\circ} \mathrm{C}$ gepaart mit starker Unruhe aufgrund von Angst oder Überforderung in der Aufnahmesituation führt in jedem Fall zu einer hohen Herzfrequenz. Daher sollte dieses Beobachtungskriterium auch immer im Zusammenspiel mit anderen Auffälligkeiten betrachtet werden. Wie in den NICE-Guidelines beschrieben, ist hier die Rekapillarisierungszeit u. a. als Ausdruck des Herzzeitvolumens ein guter Anhaltspunkt. Ist diese > 3 s, sollte ein Arztkontakt schnell erfolgen.

Dies gilt auch bei leichten Dehydratationszeichen wie trockenen Lippen oder geringer Urinausscheidung. Ist der Hautturgor schon reduziert, ist ein sofortiger Arztkontakt indiziert. Eine von den Eltern beschriebene verminderte Nahrungsaufnahme ist immer kritisch zu betrachten. Die einschätzende Person sollte immer ein Trinkangebot machen, um die Beurteilung selbst treffen zu können.

Im Verlauf der Fiebersenkung ist die Einschätzung der Aktivität von Bedeutung. Ein schlappes Kind sollte also in der engmaschigen Beobachtung des Pflegepersonals bleiben. Ein Gesichtspunkt, der bei der Beurteilung hier eine große Rolle spielt, ist die Tageszeit. Die Sorge der Eltern steigt meist zum Abend und in der Nacht. Sie beschreiben oft, 
dass die Kinder am Tage ganz agil gewesen, jetzt aber schlapp seien. Hier hilft oft ein ruhiges Gespräch über die typischen Schlafenszeiten des Kindes. Aber auch hier darf sich die einschätzende Person nicht davon täuschen lassen, um eine Fehleinschätzung zu vermeiden.

Schrilles Schreien und Berührungsempfindlichkeit sollte besonders bei Säuglingen als typisches Zeichen für eine Meningitis immer zu einem schnellen Arztkontakt führen.

Zu einem sofortigen Hinzuziehen des Arztes sollte es auf jeden Fall kommen bei einer vorgewölbten Fontanelle, bei fokalen oder generalisierten Krampfanfällen sowie anderen neurologischen Auffälligkeiten.

Grundsätzlich gilt: Ein Kind, dass von hochbesorgten Eltern vorgestellt wird mit dem Eindruck, es sei ganz anders, als sie es kennen würden, muss in die engmaschige Überwachung der Pflegekräfte, um einen Gesamteindruck über mindestens zehn Minuten zu erhalten. Jedes kritische Gefühl einer Pflegefachperson sollte zumindest eine weitere Pflegende abklären oder tatsächlich zum sofortigen Arztkontakt führen [12].

\section{ZUSAMMENFASSUNG}

- Fieber bei Kindern löst bei Angehörigen oft große Sorge aus und den Wunsch nach einer raschen Behandlung - je höher das Fieber, umso höher die Angst und das Dringen auf einen schnellen Arztkontakt.

- Es gilt, fiebernde Kinder mit besonderem Behandlungsbedarf zu identifizieren.

- Die Höhe des Fiebers allein sagt nichts über die Behandlungsdringlichkeit aus.

- Die Behandlungsdringlichkeit in der Notaufnahme muss abhängig von dem Allgemeinzustand des Kindes und der Begleitsymptomatik gemacht werden.

- Ziel ist ein patientenorientiertes, evidenzbasiertes und verantwortungsvolles Fiebermanagement im Zusammenspiel mit einer Verbesserung des Wohlbefindens des Kindes.

\section{Autorinnen/Autoren}

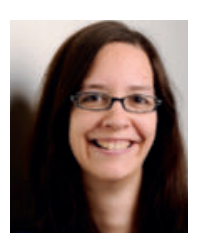

\section{Gesche Kranz}

Stellvertretende Funktionsleitung Kindernotaufnahme, Charité - Universitätsmedizin Berlin, Klinik für Pädiatrie mit Schwerpunkt Pneumologie, Immunologie und Intensivmedizin.

E-Mail: gesche.kranz@charite.de
Literatur

[1] Geissler A, Quentin W, Busse R. Umgestaltung der Notfallversorgung: Internationale Erfahrungen und Potenziale für Deutschland. In: Klauber J, Geraedts M, Friedrich J et al. (Hrsg.) Krankenhausreport 2017: „Zukunft gestalten“. Stuttgart: Schattauer; 2017, S. 41-59. Online unter bit. ly/2ZmFQmq, letzter Zugriff: 22.08.2019

[2] Behringer W, Buergi U, Christ M et al. Fünf Thesen zur Weiterentwicklung der Notfallmedizin in Deutschland, Österreich und der Schweiz. Notfall + Rettungsmedizin 2013, 16(8): 625-6

[3] Hajiloueian E. Inanspruchnahme von Notfallambulanzen in Berlin in den Jahren 2006/2007. Dissertation, Freie Universität Berlin; 2011. Online unter bit.ly/2ZkGgJW, letzter Zugriff: 22.08.2019

[4] Köster C, Wrede S, Herrmann T et al. Ambulante Notfallversorgung. Analyse und Handlungsempfehlungen. Göttingen: AQUA - Institut für angewandte Qualitätsförderung und Forschung im Gesundheitswesen $\mathrm{GmbH}$, 2016. Online unter bit.ly/2NoCPjf, letzter Zugriff 22.08.2019

[5] Christ M, Grossmann F, Winter D et al. Modern triage in the emergency department. Dtsch Arztebl Int 2010, 107(50): 892-8

[6] Krey J. Triage durch Pflegekräfte in der ZNA. Anregungen zur Übertragung internationaler Erfahrungen. Notfall + Rettungsmedizin 2007, 10(5): 329-35

[7] Fernandes C et al. Five-level triage: a report from the ACEP/ ENA Five-level Triage Task Force. J Emerg Nurs 2005; 31(1): 39-50

[8] Mackway-Jones K, Marsden J, Windle J (Hrsg.). Ersteinschätzung in der Notaufnahme: das Manchester-Triage-System. 4. überarb. u. erg. Ausg. Bern: Hogrefe; 2018

[9] Kowalzik F, Zepp F. Das fiebernde Kind. Grundlagen der Behandlung. Monatsschr Kinderheilkd 2013, 161(3): 196-203

[10] Kallinich T, Keitzer R, Puskas E et al. Exercise - induced hyperthermia in childhood: a case report and pilot study. Acta Paediatr 2009, 98(7): 1217-9

[11] Huppertz HI. Fieber ohne Fokus. Monatsschr Kinderheilkd 2013, 161(3): 204-10

[12] Niehues, T. (2013): Das fiebernde Kind: Diagnostisches Vorgehen und Behandlung. Dtsch Arztebl Int 2013, 110(45): 764-74

[13] Paes BF, Vermeulen K, Brohet RM et al. Accuracy of tympanic and infrared skin thermometers in children. Arch Dis Child 2010, 95(12): 974-8

[14] Diekmann R, Brownstein D, Gausche-Hill M. The Pediatric Assessment Triangle: A Novel Approach for the Rapid Evaluation of Children. Pediatr Emerg Care 2010, 26(4): 312-5

[15] National Institute for Health and Care Excellence. Fever in under $5 \mathrm{~s}$ : Assesment and initial management. Online unter bit.ly/2L5bTIE, letzter Zugriff 22.08.2019

\section{Bibliografie}

DOI https://doi.org/10.1055/a-1007-2780

intensiv 2019; 27: 295-300

(c) Georg Thieme Verlag KG Stuttgart · New York

ISSN 0942-6035 